\title{
Diagnosis of Oral Lesions associated with HIV/AIDS
}

Hamza O.

Department of Oral Surgery and Oral Pathology, MUHAS

Hamza O: Diagnosis of Oral Lesions associated with HIV/AIDS. Tanz Dent J 2008; 15 (1): 22 - 26

Correspondence: Hamza O, P.O. Box 15517, Dar es Salaam, Tanzania. E-mail:dromar20@yahoo.com

\section{Introduction}

Acquired Immunodeficiency Syndrome (AIDS) is caused by Human Immunodeficiency Virus (HIV). HIV is a lentivirus, subfamily of retroviruses.

HIV has a lipid envelope that has specific glycoproteins that attach to $\mathrm{CD} 4$ protein on cell surface.

Cells which express CD4 protein are at risk of infection with HIV including CD4 lymphocytes, monocytes, macrophages, microglial cells, and langerhan's cells in skin.

Disturbances in number and function of CD4 cells lead to opportunistic infections. Oral lesions (OL) are among early clinical features of HIV infection and can predict progression of HIV to AIDS.

Significance of oral lesions is that, indicates HIV infection and result in significant morbidity.

\section{Diagnosis of oral lesions}

Dental practioners (DP) may be the first to recognize the signs and symptoms of HIV. Each DP must be adept of taking medical/dental history and examining oral cavity and surrounding tissues for signs of lesions. Proper diagnosis and treatment of oral lesions have been shown to reduce the morbidity associated with HIV infection and to increase life span.

Diagnosis can be achieved after a detailed history and clinical examination of the patient. In addition, special tests in particular tissue biopsy, microbiologic investigation and hematological assessment are important in establishing definitive diagnosis.

\section{History and clinical examination}

A full medical and dental history should be taken. Specific information related to the orofacial complaint should include details of site, onset, relieving and exacerbating factors. Clinical examination should include both extra-oral and intra-oral examination. Initially an overall assessment should be made and then depending on signs and symptoms a more detailed examination of a particular area will be required.

\section{Special investigations}

These investigations depend on the oral lesions. These can be tissue biopsy, microbiologic, hematologic and salivary gland investigations. Tissue biopsy can be done in case Oral Kaposi's sarcoma, Squamous cell carcinoma, non-Hodgkin's lymphoma or tumors. Microbiologic investigations can be a smear or plain swab. Smear is obtained by spreading material scraped from lesion onto a glass slide and examined for presence of bacteria or fungi. Plain swab is used to detect bacteria, fungi and virus and sent to the laboratory for culture

\section{Classification of oral lesions}

OL associated with HIV/AIDS can be classified based on:

\section{Pathophysiological process}

EC-Clearinghouse/WHO (1993) classification (2 groups; Strongly associated with HIV and less commonly associated with HIV).

\section{Pathophysiological process: Broadly classified} into;

1 Infection (fungal, viral, bacteria)

2. Neoplasm (Kaposi's sarcoma, non-Hodgkin's lymphoma)

3. Immune-mediated (Aphthous ulcers, necrotizing stomatitis)

4. Other (xerostomia, salivary gland disease, pain syndromes)

\section{EC-Clearinghouse/WHO classification 1993 classification}

\section{Group 1: OL commonly associated with HIV}

Oral candidiasis (OC).

Oral hairy leukoplakia (OHL).

Kaposi's sarcoma (HSV type 8).

Linear Gingival Erythema -HIV related gingivitis.

Necrotizing ulcerative gingivitis.

Necrotizing ulcerative periodontitis.

Non-Hodgkin's lymphoma

\section{Group 2: OL less commonly associated with HIV}

Bacterial infections: Mycobacterium Tuberculosis, Syphilis.

Localized Bacterial infections: Sinusitis, periapical lesions, space infections, abscess and cellulitis.

Viral infections: Herpes Simplex Virus infection (HSV), Varicella-Zoster, Human Papilloma Virus (HPV) (oral warts), Cytomegalovirus (CMV), Pox virus/Moluscum contagiosum. 
Fungal: Cryptococcosis, Histoplasmosis, and Aspergillosis.

Necrotizing stomatitis/ Noma

Delayed wound healing.

Drug reactions: ulcerative, erythema multiforme,

lichenoid reactions

Non-Specific ulceration.

Recurrent Aphthous stomatitis.

Salivary gland disease: dry mouth, swelling.

Thrombocytopenia

Melanotic hyperpigmentation

Neurologic disturbances: facial palsy, trigerminal neuralgia

Squamous cell carcinoma

The frequency and incidence of these lesions will depend on a degree of immunosuppression and correlates highly with HIV progression.

OC and OHL have been incorporated in CDC clinical classification and staging of HIV disease and have been correlated with lower CD4+ count and increase in viral load

Specific Oral lesions; diagnosis and management Fungal, viral, bacterial, neoplasm, immunemediated and others

\section{Fungal infections}

Oral candidiasis

Oral candidiasis is the most common HIV-related lesion caused by Candida sp. predominantly due to C. albicans. While Candida species can be isolated from $30-50 \%$ of the oral cavities of healthy adults, making it a constituent of the normal oral flora, clinical oral candidiasis rarely occurs in healthy patients. It occurs in up to $90 \%$ of HIV/AIDS patients.

Based on clinical appearance, oral candidiasis can appear as one of four distinct clinical entities: Pseudomembranous, erythematous, hyperplastic and angular cheilitis.

\section{Pseudomembranous candidiasis}

The commonest type of OC. Appear as creamy white or yellowish patches that can be wiped off leaving a red, raw or a bleeding surface. Appears in any oral mucosa surface. Associated with initial and progressive immune deterioration.

\section{Erythematous candidiasis}

Appear as a red velvet lesion, most commonly on the palate, buccal mucosa and dorsum of tongue. Is the most commonly overlooked OC type and associated with early HIV infection.

\section{Hyperplastic/chronic candidiasis}

Appear as a white non-removable plaque/usually adherent to the tissue in any oral mucosa surface. Usually occur at a later stages of HIV infection and associated with severe immunosuppression/long standing disease. May be associated with increased risk for malignant transformation.

\section{Angular cheilitis}

Appears as cracks or fissures from the corners of the mouth, sometimes covered with pseudomembrane. Can occur during all stages of HIV infection.

\section{Diagnosis of oral candidiasis is made mainly on clinical grounds and sometimes from culture on Sabouraud Dextrose agar (SDA) and examination of $\mathrm{KOH}$ suspensions of smears.}

Treatment is must due to its local symptoms and risk of dissemination to respiratory or digestive tracts causing significant morbidity. Topical therapy is effective for limited and accessible lesions. Clotrimazole troches, nystatin pastilles and nystatin oral suspension are effective for mild to moderate OC.

Systemic treatment for OC involves the use of imidazole (ketoconazole) and triazole (fluconazole and itraconazole) antifungal medications. These can be used in case of severe or recurrent OC.

\section{Other fungal infections}

Cryptococcosis, Histoplasmosis, Aspergillosis are rare/uncommon oral deep fungal infections which require histological diagnosis

Treatment is by systemic therapy; intravenous (IV) Amphotericin B or IV fluconazole.

\section{Viral infections}

\section{OHL}

OHL is a white lesion found predominantly on the lateral margins of the tongue.

It does not rub off, frequently confused with hyper plastic OC.

Its frequency and severity is related to the degree of immunosuppression

The loss of langerhan's cells appear to be important for its development

Appears as a well-demarcated, corrugated white lesion varying from flat, plaque-like to papillaryvillous. Thought to be induced by Epstein-Barr virus (HSV type 4).

The lesion is asymptomatic however, patients may complain of appearance and fuzzy feeling. 
Diagnosis is mainly clinically; if the lesion is not apparent clinically biopsy may be indicated.

This assymptomatic lesion does not require treatment. However, for cosmetic purposes, some patients may request treatment. Oral acyclovir may be used but lesion reoccurs upon ceasing it, antiretroviral (ARVs) may be useful. Topical podophyllum resin, retinoids and surgical removal have all been reported as successful treatments.

\section{Herpes simplex infection}

Appear extra or intraorally as vesicles which later rupture to leave erosion with subsequent crust, also as ulcers. Occur more commonly on poorly keratinized tissue like the buccal mucosa and labial mucosa. Usually recurrent and painful and the associated pain can result in reduced oral intake of food and significant weight loss.

\section{HSV infection is due to reactivation of latent HSV in Trigerminal ganglion}

Diagnosis is mainly clinically, but clinical diagnosis can be assisted by viral culture and examination of a cytologic smear for the virus. Oral HSV infection responds well to systemic acyclovir.

\section{Human Papilloma Virus}

In some patients with HIV infection, HPV causes focal epithelial and connective tissue hyperplasia, forming an oral wart. Clinically 3 types of warts are seen in HIV; couliflower, spiky and flat. Oral warts are associated with increased CD4 count in patients on ARVs. Diagnosis is mainly clinically (+/- viral culture).

Surgical removal by excision, cryotherapy or Carbon dioxide laser is the treatment of choice, however, recurrence is high hence pose a significant challenge.

\section{Cytomegalovirus (CMV) infection}

Less common involving oral cavity but may cause intra-oral ulceration. However, CMV is an uncommon cause of intraoral ulceration in HIV/AIDS patients. Such lesion may represent early sign of disseminated CMV infection, which must be diagnosed as early as possible because of the serious nature of its sequelae, including retinitis and meningitis. Oral CMV appears as solitary, chronic deep ulceration most often involving the buccal and labial mucosa. Clinically, it is indistinguishable from other nonspecific ulceration. Thus for definitive diagnosis is viral culture, biopsy and histological inspection is essential.

Systemic antiviral (e.g.. Acyclovir, gancyclovir) is a treatment of choice.

\section{Herpes zoster (HZV) infection}

Usually Painful and appears as vesiculobullous lesions unilaterally.

It is caused by reactivation of Varicella zoster virus in the nerve ganglia. It affects one of the divisions of Trigerminal nerve. Diagnosis is mainly on clinical grounds.

Systemic antiviral such as acyclovir is the treatment of choice.

\section{Bacterial infections}

Although isolated cases of oral infection with Klebsiella pneumoniae, Enterobacter cloacae, Actinomyces israelii, Escherichia coli and Mycobacterium avium intracellulare have been reported in patients with HIV infection, the most common oral lesions associated with bacterial infection are periodontal diseases and less commonly are bacillary epithelioid angiomatosis and syphilis.

\section{Periodontal diseases (PD)}

In HIV associated periodontal disease (PD) the bacterial flora is no different from that of a healthy individual with PD. The clinical lesion in

HIV associated PD is a manifestation of the altered immune response to the pathogens.

HIV associated PD includes; linear gingival erythema (LGE), necrotizing ulcerative gingivitis (NUG) and necrotizing ulcerative periodontitis (NUP).

\section{LGE}

LGE can be the early sign of HIV infection and clinically characterized by distinctive red line (erythema) (1-3 mm wide) at the free gingival margin, often with petechiae. It is typically associated with no symptoms or only mild gingival bleeding and mild pain.

Differs with ordinary gingivitis that it can be seen even with excellent oral hygiene and little or no plaque accumulation. LGE unlike ordinary gingivitis, the erythema often persists following simple dental prophylaxis. Oral rinsing with Chlorhexidine gluconate $0.12 \%$, antibiotics and followed by periodontal debridement is the treatment of choice.

\section{NUP}

NUP may have same clinical presentations with those seen in immune competent, however in presence of HIV tends to be more severe, progress more rapidly and more painful and cause local necrosis of tissues. NUP is characterized by 
generalized deep osseous pain, significant erythema that is often associated with spontaneous bleeding, rapidly progressive destruction of the periodontal attachment and bone. The destruction is not selflimiting and can result in loss of the entire alveolar process in the involved area.

Treatment consist of rinsing daily with chlorhexidine gluconate $0.12 \%$, metronidazole (400 $\mathrm{mg}$ orally three times daily for 7 days) and followed by periodontal debridement and follow up.

\section{Bacillary epithelioid angiomatosis (BEA)}

This recently described lesion appears to be unique to HIV infection and often clinically indistinguishable from oral Kaposi's sarcoma (KS) since both may present as an erythematous, soft mass which may bleed upon gentle manipulation, biopsy and histological examination are required to distinguish BEA from KS. The presumed etiological pathogen, Rochalimae henselae, can be identified using Warthin-Starry staining. Both KS and BEA are histologically characterized by atypical vascular channels, extravasated red blood cells and inflammatory cells. However, prominent spindle cells and mitotic figures occur only in KS.

\section{Syphilis}

It is uncommon cause of intraoral ulceration even in HIV infection. Its appearance is no different from that observed in HIV seronegative individuals. It is a chronic, non-healing, deep, solitary ulceration often clinically indistinguishable from that due to tuberculosis, deep fungal infection or malignancy. Dark field examination may demonstrate Treponema Positive reactive plasma reagin (RPR) and histologic demonstration of Treponema pallidum is diagnostic. Patients should be referred to their physicians for evaluation and treatment. Combination treatment with penicillin, erythromycin and tetracycline is the treatment of choice.

\section{Neoplasm}

\section{Kaposi's sarcoma (KS)}

$\mathrm{KS}$ is the most common malignancy associated with HIV infection. Recognition of the lesion is essential, since oral $\mathrm{KS}$ is often the first manifestation of the disease and is a diagnostic criterion for AIDS. The lesion may appear as a redpurple macule, an ulcer or a nodule or mass. Intraoral KS occurs on heavily keratinized mucosa, often in the palate. Definitive diagnosis of $\mathrm{KS}$ requires histological examination. There is no cure for $\mathrm{KS}$, but studies have shown reduction in incidence and prevalence upon use of antiretrovirals. Chemotherapy (Intralessional vinblastine sulfate) or radiotherapy are effective with a goal being local control of size and number of lesions.

\section{Non-Hodgkin's lymphoma (NHL)}

Is the second most common malignancy in HIV after KS and is the most common lymphoma associated with HIV infection and is usually seen in late stages with $\mathrm{CD} 4$ cell count of less than $100 / \mathrm{mm}^{3}$.

It appears as rapidly enlarging mass, less commonly as ulcer or plaque and most commonly on the palate or gingiva. NHL may be indistinguishable from masses caused by $\mathrm{KS}$ or other diseases in HIV-infected patients. Histological examination is essential for diagnosis and staging.

Prognosis is poor, with mean survival time less than one year despite treatment with mult-drug chemotherapy.

\section{Immune-mediated oral lesion}

HIV infection and progression is characterized by progressive immune deterioration, it is also characterized by abnormal activation of immune system leading to tissue injury and the worsening health of the patient.

\section{Aphthous ulcers (AU)}

There three forms, Herpertiform, Minor and Major.

\section{Major apthous ulceration}

Is the most common immune-mediated HIV related oral disorder. The large solitary or multiple, chronic, deep, painful ulcerations of major aphthae appear identical to those in non-infected patients, but they often last much longer and are less responsive to therapy. Seen usually in nonkeratinized mucosa. Due to its associated pain it limits the patient dietary intake.

Diagnosis is mainly clinically, however, biopsy for histological examination may be indicated if mimic malignant disease.

Treatment requires topical corticosteroids such as clobetesol $(0.05 \%$ ointment applied for 45 seconds 3 times daily) or dexamethasone oral rinse $(0.5$ $\mathrm{mg} / 5 \mathrm{ml}$ dexamethasone elixir 3 times daily, rinse for 45 seconds and expectorate). When multiple ulcers are present or response to topical treatment is incomplete, systemic corticosteroids therapy is required (Prednisolone $1 \mathrm{mg} / \mathrm{kg}$ ).

\section{Salivary gland disease}

HIV infection is associated with salivary gland disease, characterized by gland enlargement and 
diminished flow and histologically by lymphoepithelial infiltration and benign cyst formation. Parotid gland is the mostly affected and submandibular gland rarely affected. Enlargement typically involve the tail of the parotid gland and appears unilateral but most commonly bilaterally. Appearance may rise suspicion of malignancy or infection, aspiration of a yellow mucinous secretion supports the diagnosis of HIV-related salivary gland disease.

Treatment with prednisolone, antiretrovirals therapies are suggested, rarely parotidectomy is necessary.

\section{Xerostomia}

Most often appear as a side effects of antiviral medication or antihypertensive.

Characterized by diminished salivary flow. Dryness present significant risk factor for caries, OC, mucosal injury, dysphagia and reduced food intake.

Diagnosis is clinically on the basis of little or no saliva pooling in the floor of mouth at rest; medical history of use of drug inducing xerostomia, radiotherapy also special tests such as sialography.
Treatment includes; Oral hygiene instructions, fluoride rinse, saliva substitutes and more fluids intake.

\section{Other lesions}

\section{Mucosal hyperpigmentation}

Have been reported in $2-5 \%$ of HIV-infected patients.

May be due to medications taken such as zidovudine, adenocortical destruction or as a direct result of the virus. Appear as well defined brownblack macules.

\section{Recommendations}

Oral pain or discomfort can lead to problems with eating hence reducing nutrients intake in HIVinfected patients hence increased the morbidity. One of the effective ways to deal with HIV/AIDS is to maintain good nutrition through early diagnosis and management of HIV-associated oral lesions.

Thus oral health care (OHC) is important and there is a need to integrate $\mathrm{OHC}$ with the general care of these patients. There is a need for training oral health care workers on diagnosing and managing oral manifestations of HIV, this will facilitate early diagnosis of HIV infection and proper management of patients.

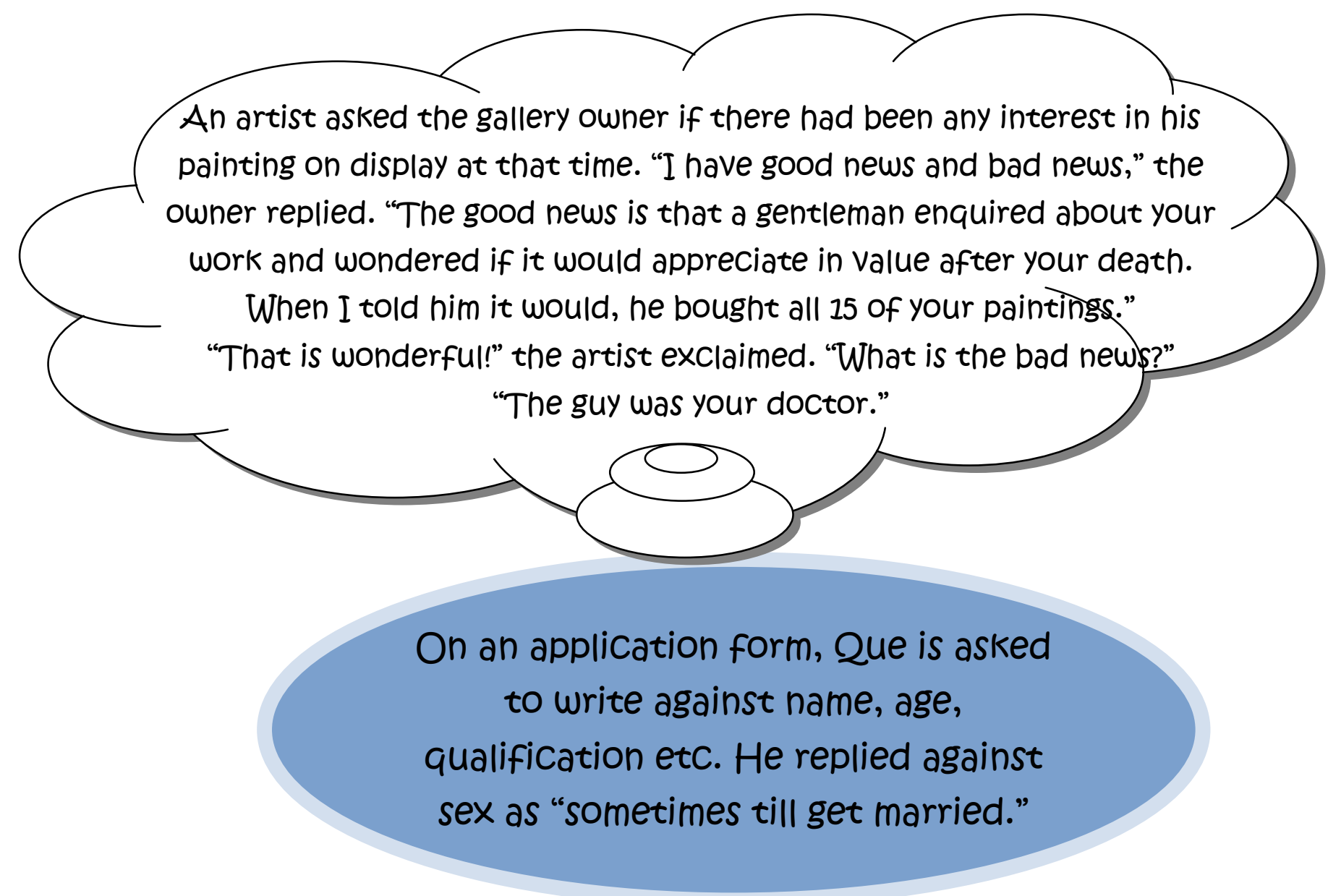

